\title{
HIPPARCOS PHOTOMETRY OF MINOR PLANETS
}

\author{
B. Morando ${ }^{1}$, F. Mignard ${ }^{2}$, \\ ${ }^{1}$ Bureau des Longitudes / URA CNRS 707, \\ 77 Av. Denfert Rochereau, F75014 Paris, FRANCE \\ ${ }^{2}$ Observatoire de la Côte d'Azur / URA CNRS 1360 / CERGA, \\ Av. Copernic, F06130 Grasse, FRANCE
}

ABSTRACT. In addition to its astrometric capabilities the Hipparcos main detector proves to be a good phototometer. The main features of the photometric reduction applied to minor planets are outlined. The apparent magnitude measured by Hipparcos is transformed into absolute magnitude after corrections for the distance to the sun, the earth and for the phase effect, are applied. We show that the remaining signal contains information on the rotational properties of the planets.

\section{INTRODUCTION}

The Hipparcos satellite launched by ESA on August 1989, has been primarily designed as a powerful instrument to carry out space astrometry at the milliarcsecond level. However manufactured as an instrument working by counting photons entering the telescope, Hipparcos has the capability of measuring star brightness as well. Thus any stretch of starlight recorded by Hipparcos is analysed in view of extracting the photometric information in addition to the positional's.

This photometry is essentially achieved by counting the number of photons crossing the entrance pupil per unit of time and collected by the detector located behind the main grid. Then these numbers are converted into a magnitude defined by a set of standard stars and a model of the instrument response. Details on this processing are available in Mignard et al., 1992-a, 1992-b, along with a summary of the results already obtained on single and double stars. In the following we take up the problem of measuring the magnitude of the 48 minor planets regularly observed in the Hipparcos program. However we start with a brief summary of the Hipparcos photometric processing, which applies without modification to stars and planets. Then we proceed to the specific corrections to be applied to the apparent magnitude of the planets to extract relevant information on the physics of these bodies.

\section{THE PHOTOMETRIC SIGNAL}

About every $2 s$ the photons counts for each star observed are fitted to a five parameter model with two harmonics. The global signal $S(t)$ is,

$$
S(t)=I+B+I M \cos (\omega t+\phi)+I N \cos (2 \omega t+\psi)
$$

where $I$ is the total intensity, $B$ the unmodulated background, $M$ and $N$ the modulation coefficients of the first and second harmonic respectively and $\phi$ and $\psi$ the corresponding phases. For a pointlike source we have typically $M=0.72$ and $N=0.25$. Thus the photometric information appears in $I, I M$ and $I N$ and must be extracted from the signal. Once this is done the Hipparcos magnitude is computed with the usual definition

$$
H_{p}=-2.5 \log _{10} \frac{I}{I_{0}}
$$


where $I_{0}$ is an arbitrary reference intensity. In the following this reference is chosen such that

$$
H_{p}=0 \equiv I_{0}=7 \times 10^{6} \mathrm{~Hz}
$$

However this is completely arbitrary and just a convenient number put into the software which allows the Poisson photon noise to be readily evaluated. The actual definition lies in the computation of the H-Magnitude for a well defined set of stars, referred to as the photometric standard stars (Grenon et al., 1992).

The typical background noise is of the order of $40 \pm 10 \mathrm{~Hz}$, depending mainly on the galactic latitude of the observation and on the satellite altitude. Its average value over a full revolution of the satellite about its spin axis ( 2.13 hours) is well determined, whereas fluctuations about the mean are poorly ascertained. As for the observation time allocation $t$, in standard conditions, we have four stars in the field of view crossing the grid. Therefore one can allocate about $4 \mathrm{sec}$ of observation per transit for an average star of magnitude 8. A larger allocation of $\sim 12 \mathrm{sec}$ is available for fainter stars and a smaller $\sim 1 \mathrm{sec}$ for the brightest. The detector can track the path of one star at a time, but a computer controlled device allows to sample light of all program stars in the field of view during their passage across the field. At this level the observation strategy makes no difference between a star and a minor planet. The latter is considered as a faint star, except Ceres.

Each star or planet transiting on the Hipparcos grid results in one determination of the magnitude ; for a given object, observations are repeated at different intervals ( $20 \mathrm{~min}$, 2 hours, 3 weeks etc...) over the mission duration, the peculiarities of which are tied to the Hipparcos unusual scanning law. A careful instrumental calibration performed every ten hours is used to get rid of the sensitivity variations with the image location and of the effects brought about by the aging of the instrument. This in turn permit to combine observations performed at very different times. Altogether an average object is observed over about 150 transits during the Hipparcos mission. The precision obtained per transit are shown in table 1 as a function of the star magnitude.

Table 1. Photometric precision for one field transit and after one year of data collection.

\begin{tabular}{lcccccccccc}
\hline Magnitude & $<4$ & $4-5$ & $5-6$ & $6-7$ & $7-8$ & $8-9$ & $9-10$ & $10-11$ & $11-12$ & $12-13$ \\
\hline Precision (0.001 mag) & 2 & 4 & 4 & 7 & 10 & 14 & 17 & 30 & 45 & 70 \\
Precision (0.001 mag) & 1 & 1 & 1 & 1.5 & 2 & 3 & 4 & 7 & 12 & 25 \\
\hline
\end{tabular}

The processing of one year of data has shown that the above figures are realistic, and provided systematic errors can be kept at such a low level that one can benefit from a tremendous statistical improvement by combining observations of constant stars into a single, better defined, magnitude. For those stars we obtain a precision of 0.001 magnitude for a 6 mag star and 0.004 for a 10 mag star. It is not clear at the moment whether we have come close to the limit as noise not related to photon noise will take over and prevent from improving the determination. However the main interest of having a reliable and stable photometry over the misison duration is the study of the short term variability in particular when the object can be viewed repeatedly for several days before the scanning moves on across the sky. This description applies without change to minor planets. One must keep in mind that apart Ceres, most of the planets have an apparent magnitude 
larger than 10, and often as large as 12 . So the accuracy is rather limited, at the level of the transit. Due to the expected variation with the planet rotation over duration as short as one hour, one cannot combine this individual measurement into a single one of better statistical quality, contrarily to the stars.

\section{ABSOLUTE MAGNITUDES}

In order to obtain informations on the physical properties of the surfaces of the planets involved, the determined magnitudes should be corrected for phase as well as for distances to the sun and to the earth. According to a decision taken by the International Astronomical Union in 1985 the apparent magnitude of a minor planet should be computed by Bowell's formula (Tansactions of the IAU, 1985) :

$$
m=5 \log (r \Delta)+H-2.5 \log \left((1-G) \Phi_{1}+G \Phi_{2}\right)
$$

where :

$$
\Phi_{i}=\exp \left\{-A_{i}\left[\tan \left(\frac{\beta}{2}\right)\right]^{B_{i}}\right\} \quad i=1,2
$$

with :

$$
A_{1}=3.33 \quad A_{2}=1.87 \quad B_{1}=0.63 \quad B_{2}=1.22
$$

In this formula $r$ is the distance of the planet to the sun, $\Delta$ the distance of the planet to the earth, $\beta$ the phase angle and $H$ is called the absolute magnitude of the planet. The values for $G$ are published annually in the Ephemerides of minor planets.

For each value of the time for which an observed value $m$ of the magnitude was obtained, $r, \Delta, \beta$ were computed from the ephemerides (Bec-Borsenberger, 1990) and formula (3) gave the corresponding value of $H$.

\section{LIGHT CURVES FOR SOME PLANETS}

For many of the observed minor planets several values of the abolute magnitudes within time intervals of a few hours were obtained. As the rotation periods of these bodies are typically 5 to 9 hours, significant parts of the light curves could thus be constructed and compared to the theoretical curves.

Let the aspect $\xi$ be the angle of the direction planet-earth with the rotation axis of a three axes ellipsoid planet whose semi-axes are $a, b, c$. Then the amplitude of the light curve due to the rotation of the planet, assuming that the albedo of the surface is constant, is given by the following expression (Binzel et al., 1989) :

$$
2.5 \log \frac{a}{b}-1.25 \log \left(\frac{a^{2} \cos ^{2} \xi+c^{2} \sin ^{2} \xi}{b^{2} \cos ^{2} \xi+c^{2} \sin ^{2} \xi}\right)
$$

If $a$ is equal to $b$, that is if the planet is axi-symetrical about its axis of rotation, the amplitude of the light curve is zero unless there are albedo variations on the surface.

Figure 1 shows the light curve of 1-Ceres for 100 days. This planet being spherical with an even surface the absolute magnitude $H$ is practically constant, the discrepancies being of the order of the rms on the determination of the magnitude. 


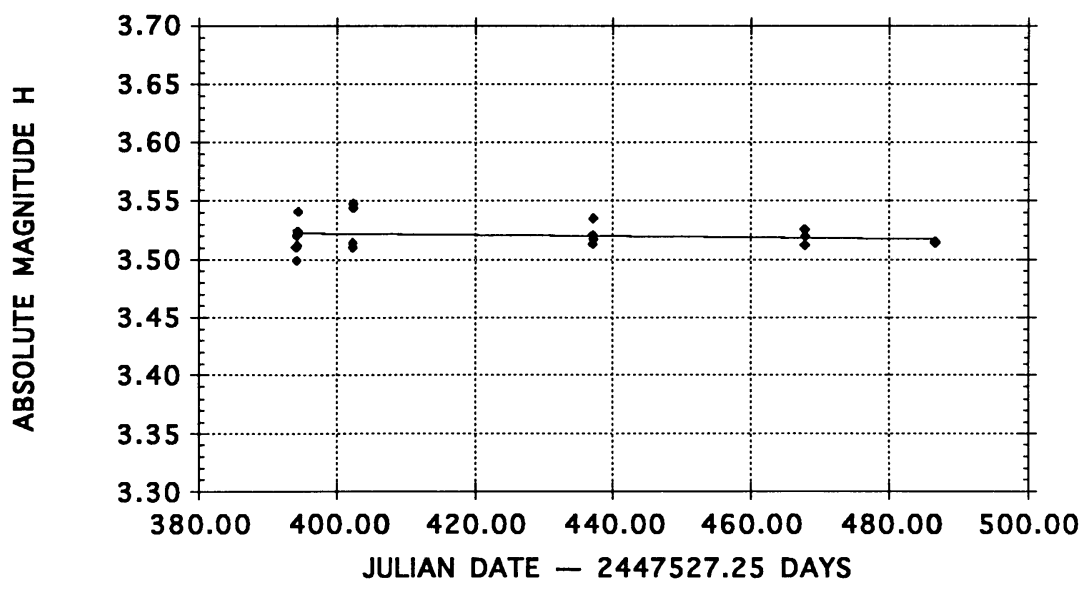

Fig.1 Variation of the absolute magnitude of Ceres in 100 days

On fig. 2 is shown the light curve of 4 -Vesta during 4.3 hours, which is nearly a period (the period is 5.342 hours). For Vesta $a$ is equal to $b$ so that the light curve should be flat, and yet an 0.1 magnitude variation largely exceeding the estimated rms is clearly visible.

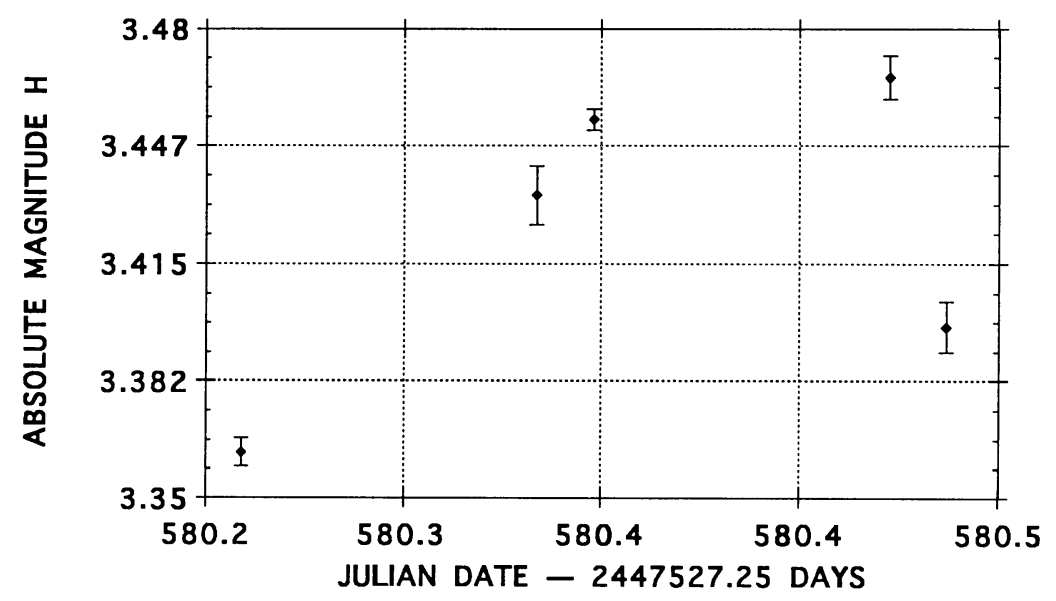

Fig.2 Variation of the absolute magnitude of Vesta in 4.3 hours 
This could be explained by the fact that the albedo of Vesta is far from being constant, the existence of a large black patch being suspected from ground based observations that show an amplitude of the light curve due to this phenomenon equal to 0.12 magnitude (Degewij et al., 1979).

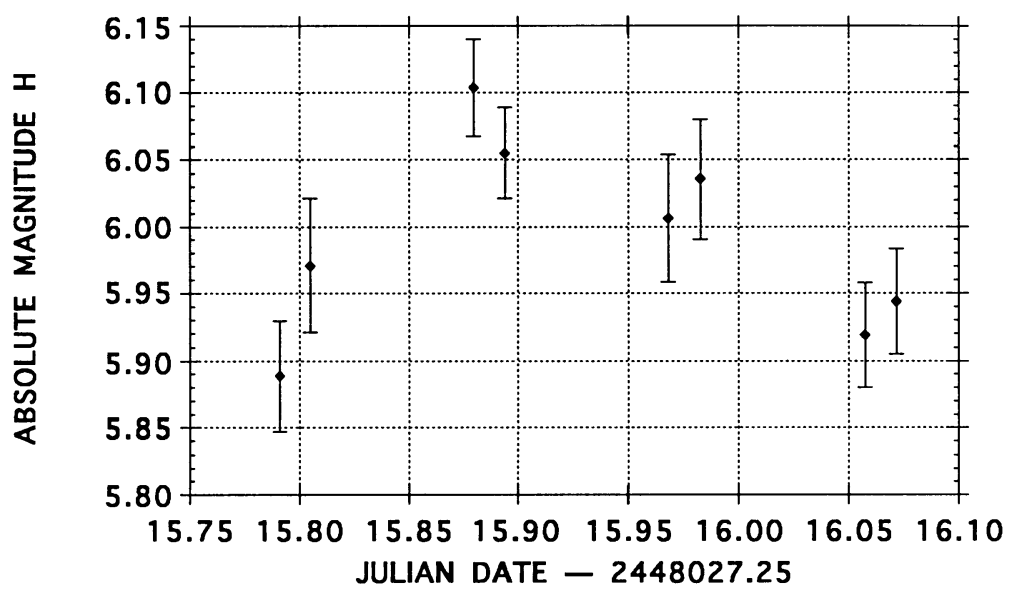

Fig.3 Variation of the magnitude of Hebe in 6.7 hours

The rotation period of minor planet 6 -Hebe is 7.274 hours, $a / b$ is equal to 1.24 and $b=c$. During the 6.7 hours registered on fig. 3 , the aspect was equal to $64^{\circ}$ so, according to formula (4), the light curve should be a smooth sinusoid with an amplitude of 0.24 magnitude. Yet, looking at Fig. 3 and in spite of the amplitude of the errors, the reader may find that the actual variations are perhaps not so simple

Other, some times unexpected, variations of the magnitude were observed for other planets. It was shown also that for different values of the aspect $\xi$ the amplitude of the magnitude variations are different. Thus for 349-Dembovska $a / b=1.28$ and $b / c=1.15$. Its magnitude varies between 5.6 and 6.3 when $\xi=92^{\circ}$ (the phase angle is then equal to $14^{\circ}$ ), but when $\xi$ is $98^{\circ}$ the phase angle is $17^{\circ}$ and the absolute magnitude varies between 5.35 and 6.45 . Such a behaviour could, of course, have been expected but the values thus found make up a valuable set of data.

\section{CONCLUSION}

The determination of the magnitudes of the minor planets using the modulation coefficients given by the Hipparcos observations seems to give sufficiently precise and reliable results. Used to reconstruct the behaviour of the absolute magnitude during the rotation of the planet about its axis, these data give significant informations which, when carefully studied, will improve the knowledge of the physical properties of the minor planets. Yet, in order to do so, one should have at one's disposal a sufficiently dense set of magnitude measurements made in a time interval of a few hours, which happens often enough when the planet is observed in the two fields of Hipparcos on successive scanning circle. 
Moreover it will be interesting to get such sets of observations for different values of the aspect $\xi$. This goal can be reached as the mission extends over several years.

\section{REFERENCES}

Bec-Borsenberger A., 1990, Ephemerides of the 48 Hipparcos minor planets, A\&AS 86, 299-349

Binzel R. P., Gehrels T., Matthews M. S. 1989, Asteroids II, University of Arizona Press

Ephemerides of minor planets for 1992, Institute of theoretical astronomy of the Russian Academy of sciences, Naouka, St Petersburg

Degewij J., Tedesco E. F., Zellner B. 1979, Icarus 40, 364-374

Grenon M., Mermilliod M., Mermilliod J. C. 1992, The Hipparcos Input Catalog. III Photometry, A\&A 258, 88-93

Mignard F., Froeschlé M., Falin J.-L. 1992-a, Hipparcos photometry : FAST main mission reduction, A\&A 258, 142-148

Mignard F., Froeschlé M., Badiali M., Cardini D., Emanuele A., Falin J.-L., Kovalevsky J. 1992-b, Hipparcos double stars recognition and processing with the FAST consortium, A\&A 258, 165-172

Transactions of the International Astronomical Union. 1985, Reidel XIXB, 184-185 